\title{
Developing patient-centred strategies to optimize the management of vasomotor symptoms in breast cancer patients: a survey of health care providers
}

\author{
Katherine Marie Cole ${ }^{1} \cdot$ Mark Clemons $^{1,2} \cdot$ Meshari Alzahrani $^{1} \cdot$ Gail Larocque $^{3} \cdot$ Fiona MacDonald $^{3}$. \\ Lisa Vandermeer ${ }^{2} \cdot$ Brian Hutton $^{4} \cdot$ Ardelle Piper $^{5} \cdot$ Greg Pond $^{6} \cdot$ Sharon McGee ${ }^{1,2}$ (1)
}

Received: 3 February 2021 / Accepted: 10 March 2021 / Published online: 22 June 2021

(c) The Author(s), under exclusive licence to Springer Science+Business Media, LLC, part of Springer Nature 2021

\begin{abstract}
Purpose Vasomotor symptoms (VMS) such as hot flashes and night sweats are common in breast cancer patients and can affect both quality of life and treatment adherence. However, there is limited practical data to guide clinicians in the optimal selection of therapeutic strategies. A survey of health care providers was performed to better understand perspectives and prescribing practices for managing this problem.

Methods Canadian health care providers who treat patients with early stage breast cancer (EBC) participated in an anonymous electronic survey. Participants provided their perspectives on the prevalence and severity of VMS among patients with EBC, outlined their management strategies, and provided feedback on the perceived efficacy of interventions for VMS. Results Responses were received from 65 providers including breast oncologists $(36 / 65,55 \%)$ and nurses with oncology expertise $(29 / 65,45 \%)$. Seventy-seven percent of participants reported regularly asking patients about VMS, and most indicated that bothersome VMS occurred in the majority of patients. Health care providers cited hot flash severity and sleep disruption as the most important issues for patients. The most common first- and second-line interventions recommended were lifestyle modifications $(n=32 / 65,49.2 \%)$ and pharmacologic strategies $(n=27 / 65,41.5 \%)$, respectively. Most respondents felt that interventions, including pharmacologic, over-the-counter, and complementary therapies, were only "somewhat effective". Overall, half of respondents $(n=35 / 65,54 \%)$ reported being "confident" in managing VMS.

Conclusion Given the variability of treatment recommendations, and health care provider uncertainty around the benefits of therapies for VMS, more 'real-world' trials are needed to optimize patient care.
\end{abstract}

Keywords Breast cancer $\cdot$ Hot flashes $\cdot$ Vasomotor symptoms $\cdot$ Survivorship

Sharon McGee

shmcgee@toh.ca

1 Division of Medical Oncology, Department of Medicine, The University of Ottawa, 501 Smyth Road, Ottawa, ON K1H 8L6, Canada

2 Cancer Therapeutics Program, The Ottawa Hospital Research Institute, Ottawa, ON, Canada

3 The Ottawa Hospital Cancer Centre, Ottawa, ON, Canada

4 Clinical Epidemiology Program, The Ottawa Hospital Research Institute, Ottawa Ontario, Canada

5 University of Ottawa Health Services, Ottawa Ontario, Canada

6 Department of Oncology, McMaster University, Hamilton, ON, Canada

\section{Background}

Vasomotor symptoms (VMS) such as hot flashes and night sweats are common in breast cancer patients, and can occur as a consequence of natural menopausal and/or anti-cancer therapies. Over $30 \%$ of post-menopausal women [1] and up to $95 \%$ of pre-menopausal women will experience VMS as a result of breast cancer treatments [2]. Despite the frequency of this problem, one-third of patients report that health care providers do not routinely ask them about VMS during regular follow up visits [3]. Furthermore, given the negative impact of VMS on quality of life (QoL) [4], untreated hot flashes and night sweats contribute to treatment discontinuation in up to $25 \%$ of patients, with detrimental impacts on breast cancer recurrence risk, and overall survival [5]. Effective management of VMS in patients with early stage 
breast cancer (EBC) is therefore a critical, but frequently unmet need.

While estrogen replacement therapy is the most effective treatment for VMS symptoms in the general population [6], it is relatively contraindicated in breast cancer patients, particularly those with estrogen responsive disease [7]. Other interventions used to manage VMS in EBC patients include lifestyle modifications (e.g. exercise, smoking cessation, use of fans, dressing in layers), non-prescription supplements (e.g. Vitamin E, black cohosh), complementary therapies (e.g. acupuncture) and prescription medications (e.g. gabapentin or venlafaxine). We recently conducted a systematic review and meta-analysis of pharmacological and non-pharmacological strategies for the management of VMS in EBC patients. The review identified 40 studies, many of which were small and of poor methodological quality, with highly heterogeneous patient populations and variable endpoints studied [8]. Moreover, there were limited randomized studies directly comparing active interventions. Thus, while we identified several treatments that might be effective in managing VMS, we did not identify a single optimal treatment strategy [8].

To aid the development of future randomized, pragmatic, patient-centred clinical trials on this subject, we surveyed Canadian health care providers, to determine their experience of VMS amongst breast cancer patients, recommendations for management, and the perceived efficacy of these interventions.

\section{Materials and methods}

\section{Study population}

Canadian health care providers who treat, or participate in the management of EBC patients were surveyed. Eligible health care providers included medical oncologists, radiation oncologists, surgical oncologists, general practitioners in oncology, nurse practitioners, and oncology nurses specializing in breast cancer.

\section{Study outcomes}

The two major objectives of this survey were to: 1 ) determine health care provider perspectives on the prevalence and severity of VMS 2) identify common management strategies including prescribing practices and their perceived effectiveness.

\section{Survey development}

This survey was developed by physicians, nurses, and researchers with expertise in both breast cancer management and survey development. The electronic survey consisted of one question to assess eligibility, one question to determine provider specialty, and 13 additional multiple-choice questions divided into two major sections. (Online resource 1) The first section asked respondents for their perspective on the prevalence of VMS in EBC patients, and what they perceived as the most bothersome symptoms for patients. Providers were also asked to indicate their clinical "trigger" for initiating therapy for the management of VMS. In the case of providers who were not directly responsible for prescribing treatments, participants were asked to provide their perspective on witnessed prescribing practices. In the second section, providers were asked to indicate their preferences for managing VMS including lifestyle modifications, alterations in cancer therapies, or use of pharmacologic, over-the-counter, and/ or complementary therapies. Respondents were also asked to comment on the perceived effectiveness of these interventions, and their overall confidence in their ability to effectively manage VMS in EBC patients. Prior to survey dissemination, the survey was pilot tested on three oncologists, one advance practice nurse, and two non-healthcare professionals.

\section{Survey implementation}

Physicians from across Canada were invited to participate in an electronic survey through a collection of publicly available email addresses used by the research team in previous surveys [9] This includes members of the Canadian Association of Medical Oncologists and the Canadian Association of Radiation Oncologists. Oncology nurses affiliated with The Ottawa Hospital Cancer Centre were approached directly to participate in the survey by study team members. Additional nursing participants were also invited to participate through the Canadian Association of Nurses in Oncology (CANO) membership email pool. Participants received a survey invitation, a survey link, and a study information sheet. A reminder notice was sent out to all potential participants two weeks after the initial invitation. Participants completed the survey through Microsoft Forms, which was available on a protected server at the Ottawa Hospital Research Institute. Completion of the survey implied consent to participate. This survey was approved by the Ontario Cancer Research Ethics Board (OCREB).

\section{Sample size}

Our goal was to obtain responses from 50 health care providers. With 50 respondents, a 95\% confidence interval would have maximum width of $<0.30$, which was deemed sufficiently precise to obtain reasonably accurate estimates of interest. Assuming a response rate of $30 \%$, we intended to reach out to 175 providers.

\section{Data analysis}

Descriptive statistics and graphics were analysed and created using Microsoft Excel. The frequency of each answer 
choice was tabulated as a proportion of the total number of respondents for that category.

\section{Results}

\section{Survey participants}

From June 16 to October 26, 2020, survey invitations were sent nationwide to 249 breast cancers specialists ( 135 medical oncologists, 114 radiation oncologists), fifty-one oncology nurses at The Ottawa Hospital Cancer Centre (TOHCC),

Table 1 Baseline Provider Characteristics

\begin{tabular}{lll}
\hline Profession & $\mathrm{N}$ & $\mathrm{N}(\%)$ \\
\hline & 36 & \\
Physicians & & \\
Medical Oncology & $21(58.3 \%)$ \\
Radiation Oncology & $12(33.3 \%)$ \\
General Practitioner in Oncology (GPO) & $1(2.8 \%)$ \\
Other, please specify & \\
Surgical Oncology & $1(2.8 \%)$ \\
General Internist & $1(2.8 \%)$ \\
Nurses and Physician Assistants & \\
Nurse Practitioner & 29 & $3(10.3 \%)$ \\
Oncology Nurse & & $24(82.8 \%)$ \\
Clinical Trial Nurse in Oncology & $1(3.4 \%)$ \\
Breast Cancer Patient Educator & & $1(3.4 \%)$ \\
\hline
\end{tabular}

and the complete membership list of CANO. Of the physicians invited, 37 could not be reached and were deemed ineligible (26 out-of-office replies, and 11 undeliverable emails). Of the remaining 212 physicians, 36 individuals completed the survey, for a response rate of $17 \%$. The membership list for CANO does not differentiate members by the type of patients they treat and therefore neither a denominator nor a response rate for this group is possible.

The participants consisted of 36 physicians (55\%) and 29 nurses (45\%) (Table 1.) Of the physician respondents, the majority were medical oncologists $(n=21 / 36,58 \%)$, or radiation oncologists $(12 / 36,33 \%)$, with one surgical oncologist, one general internist, and one general practitioner in oncology (GPO) completing the survey. Of the nursing respondents, the majority were nurses with experience in oncology practice $(24 / 29,83 \%)$, with the rest comprising nurse practitioners (3/29), one clinical trials nurse, and one patient educator.

\section{Vasomotor symptoms: prevalence and severity}

Health care providers were asked how often they assess VMS. The two most common response were "every visit following initiation of a new endocrine therapy/ovarian function suppression" (28/64, 43.8\%), and at "every visit" $(21 / 64,32.8 \%)$ (Table 2$)$ Sixty percent $(n=39 / 65)$ of respondents indicated that bothersome hot flash symptoms were experienced by the "majority" of their patients, while $26 \%$ felt it was an issue for a "minority" of patients ( $n=17 / 65$ ) (Table 2.) When asked to rank what they perceived were the most bothersome VMS experienced by patients, the answers were; sleep disruption $(26 / 65,40 \%)$,

Table 2 Frequency of Hot Flash Assessments and Magnitude of the Problem

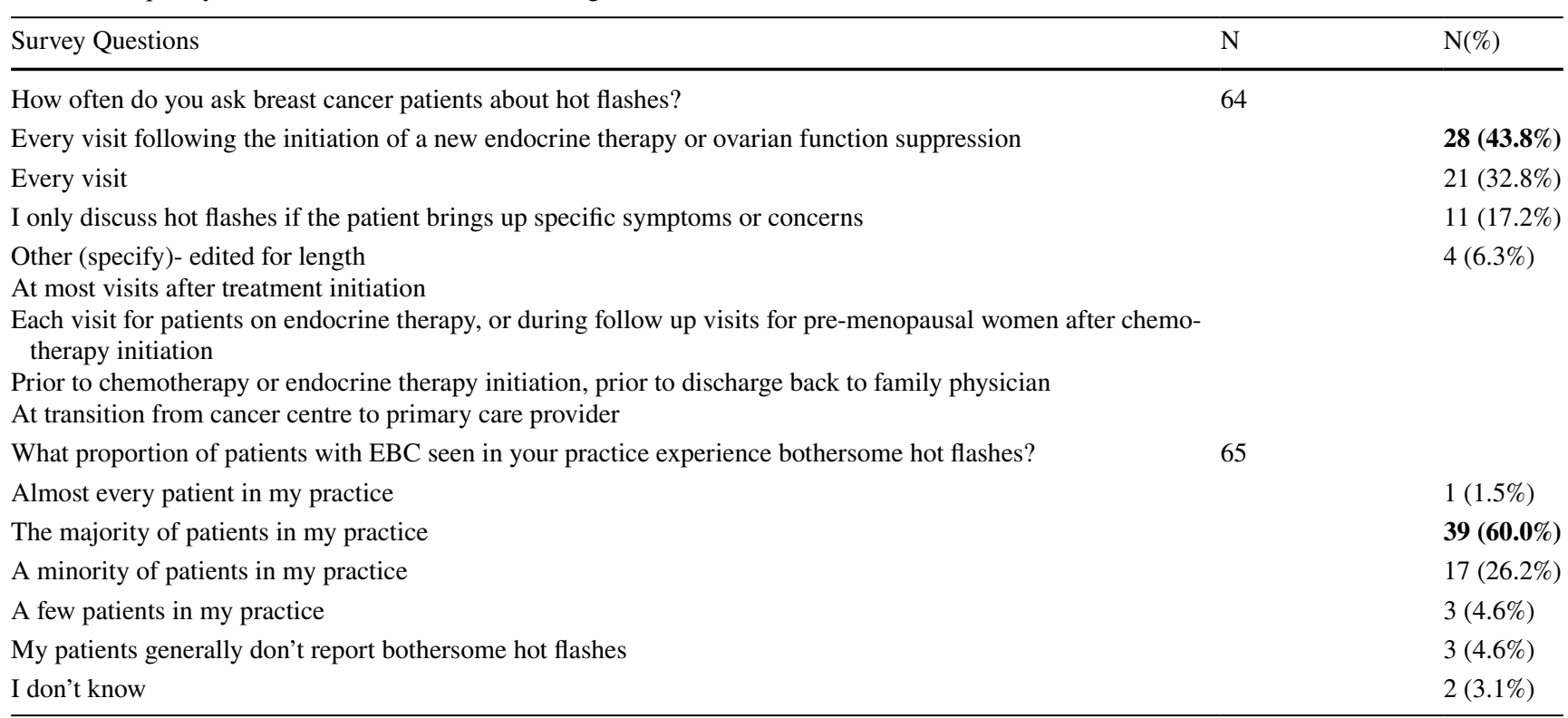

Numbers in bold represent majority response 
hot flash severity $(23 / 65,35.4 \%)$ or hot flash frequency $(8 / 65,12.3 \%)$ (Online resource 2$)$. Conversely, the majority of providers thought that the least bothersome symptom for patients were concerns around intimacy $(43 / 65,66 \%)$.

Multiple indications to start interventions for VMS were identified (Fig. 1). These included: when "the frequency/severity of their hot flashes were bothersome" ( $n=44 / 65,67.7 \%$ ), "the patient was considering discontinuation of their cancer treatment" $(n=40 / 65,61.5 \%)$, "they specifically request an intervention for their hot flashes" ( $n=37 / 65,56.9 \%)$, or "they were having persistent sleeping difficulties" ( $n=34 / 65,52.3 \%)$. Other indications for starting interventions included: when patients "were having persistent difficulties working/performing their activities of daily living" $(n=30 / 65,46.2 \%)$, or "their mood symptoms were unmanageable" $(n=27 / 65,41.5 \%)$.

\section{Management strategies}

For the majority of respondents $(n=32 / 65,49.2 \%)$ the most common interventions utilized in the $1^{\text {st }}$ line were lifestyle modifications. ( $n=32 / 65,49.2 \%$ ) (Fig. 2). The most common 2nd line interventions were pharmacologic strategies $(n=27 / 65,41.5 \%)$, and the most common 3rd interventions were cancer treatment modifications $(n=17 / 65,26.2 \%)$, and pharmacologic interventions $(n=16 / 65,24.6 \%)$.

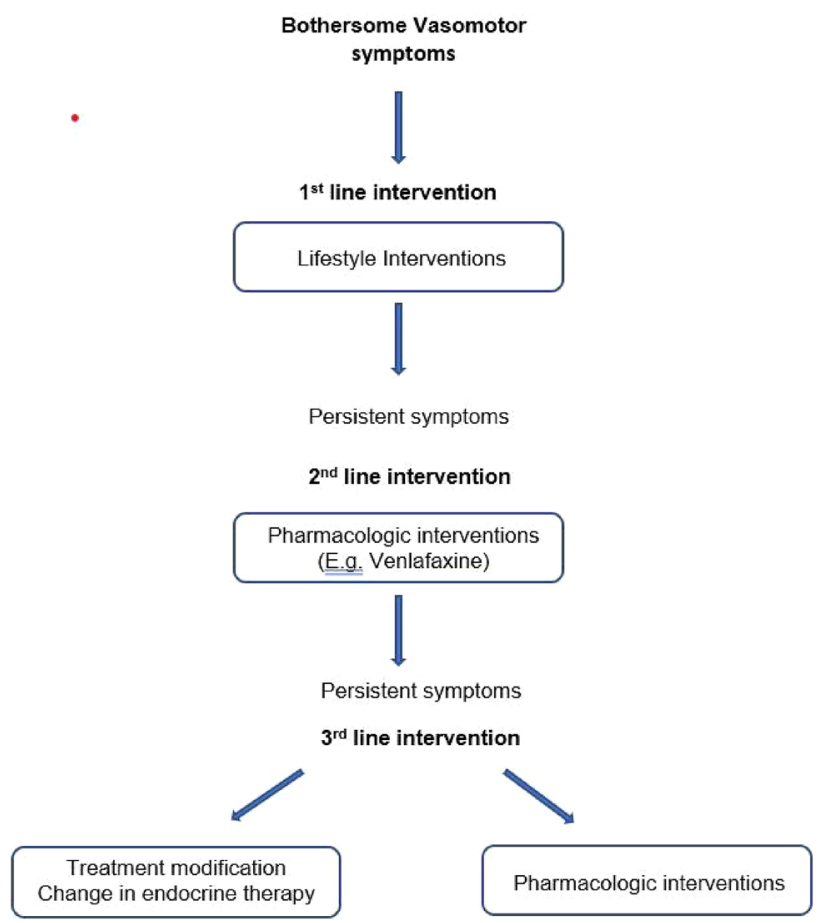

Fig. 2 Flow diagram depicting most common interventions for vasomotor symptoms in early breast cancer patients as selected by Canadian health care providers

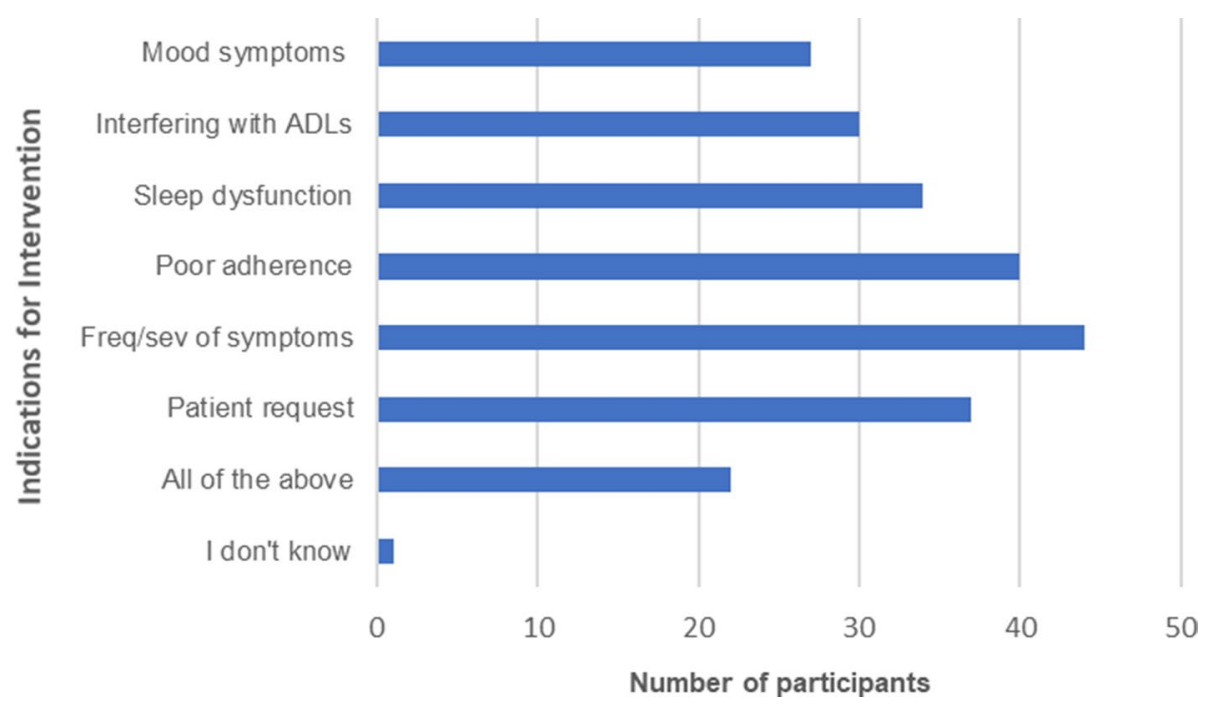

Fig. 1 Indications for initiating interventions for vasomotor symptoms in early stage breast cancer patients. Participants were prompted to select one or more options including "the frequency/severity of their hot flashes was unmanageable", "the patient was considering discontinuation of their cancer treatment due to their hot flashes or adherence to treatment was inconsistent due to hot flashes", "they were having persistent sleeping difficulties", "they were having persistent difficulties working/performing their activities of daily living" (ADLs), "their mood symptoms were unmanageable", "I don't know", "all of the above", and "other" (please specify) 
Table 3 Interventions for Hot Flashes in Any Line

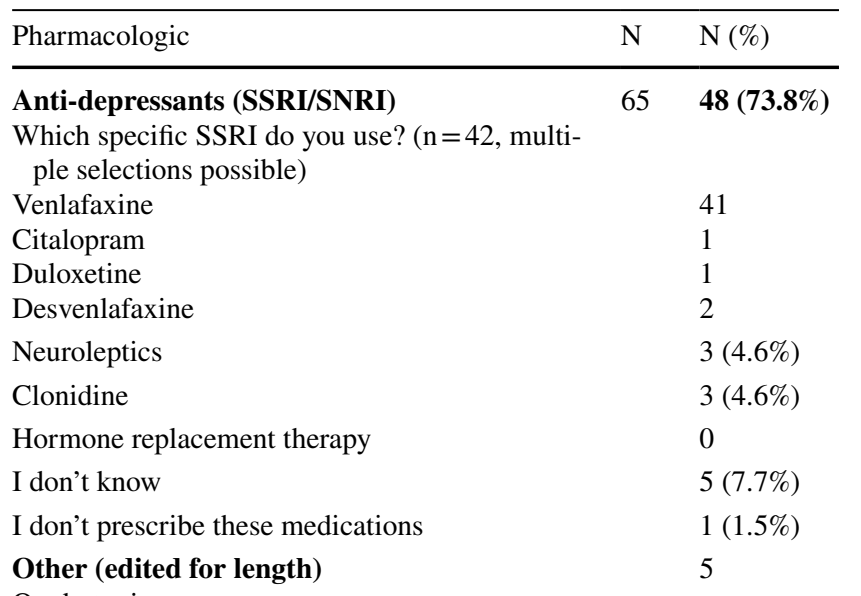

Oxybutynin

Stop endocrine therapy if low risk breast cancer

Treatment break

Approach is provider dependent

Gabapentin if insomnia, otherwise, venlafaxine

Over-the-counter supplements

Vitamin E (oral/vaginal)

Black cohosh

Soy/milk protein

Melatonin

Red clover

Evening primrose oil

I don't know

I don't typically make these recommendations

Complementary therapies

Acupuncture

Relaxation therapy

Exercise therapy/yoga

CBT or counselling

Hypnosis

I don't know

I don't typically make these recommendations

Other

Refer to support therapist/psychologist

Anti-cancer Treatment modification

Dose reduction

Treatment break

Change of endocrine therapy

Stop endocrine therapy

I don't know

I don't typically make these recommendations

Other (Specify) (edited for length):

Dose reduction or change therapy (2)

Numbers in bold represent majority response

0

$5(8.1 \%)$

0

64

$39(62.9 \%)$

$12(18.5 \%)$

$3(4.6 \%)$

$21(32.3 \%)$

$2(3.1 \%)$

0

$2(3.1 \%)$

$23(35.4 \%)$

$1(1.5 \%)$

65

$1(1.5 \%)$

$2(3.1 \%)$

$5(7.7 \%)$

\section{Pharmacologic strategies}

The most commonly utilized pharmacologic strategies were anti-depressants $(48 / 65,73.8 \%)$. The most selected agent was venlafaxine $(n=41 / 42,97.6 \%)$, other agents like citalopram (1/42), duloxetine (1/42) and desvenlafaxine $(n=2 / 42)$ were rarely selected. (Table 3$)$. When asked how effective pharmacologic interventions are in managing VMS, $83 \%$ of health care providers reported them it to be "somewhat effective". $(n=54 / 65)$ (Table 4$)$.

\section{Non-prescription supplements and complementary therapies}

When asked about commonly recommended over-thecounter supplements, most respondents $(39 / 62,62.9 \%)$ indicated that they do not routinely recommend these agents (Table 3). Other responses included; black cohosh $(8 / 62)$, vitamin E (5/62), evening primrose oil $(5 / 62)$, and melatonin (4/62). Over-the-counter/supplement therapy were deemed to be "somewhat effective" by $42 \%$ of respondents $(n=27 / 65)$, with $26 \%$ of respondents

Table 4 Perceived Overall Effectiveness of Therapy

\begin{tabular}{lll}
\hline & $\mathrm{N}$ & $\mathrm{N}(\%)$ \\
\hline Pharmacologic Strategies & 65 & \\
Very effective & & $5(7.7 \%)$ \\
Somewhat effective & & $\mathbf{5 4}(\mathbf{8 3 . 1 \% )}$ \\
Not effective & $1(1.5 \%)$ \\
I don't know & $2(3.1 \%)$ \\
I have never prescribed & & $3(4.6 \%)$
\end{tabular}

Over-the-counter supplements

65

Very effective

$2(3.1 \%)$

Somewhat effective $\quad 27(\mathbf{4 1 . 5 \% )}$

Not effective $11(16.9 \%)$

$\begin{array}{lll}12(18.5 \%) & \text { I don't know } & 8(12.3 \%) \\ 10(15.4 \%) & \text { I have never prescribed } & 17(26.2 \%)\end{array}$

33(50.8\%) Complementary therapies 65

Very effective

$1(1.5 \%)$

Somewhat effective

$30(46.2 \%)$

Not effective

$7(10.8 \%)$

$\begin{array}{ll}\text { I don't know } & 11(16.9 \%) \\ \text { I have never recommended these treat- } & 16(24.6 \%)\end{array}$ ments

Numbers in bold represent majority response 
indicating that they have never recommended these treatments, $17 \%$ indicating that they are "not effective" and $12 \%$ answering "I don't know" (Table 4). When providers were asked about commonly recommended complementary therapies, the most common response was "I don't typically make these recommendations" ( $n=23 / 64$, $35.4 \%$ ) (Table 3.). Other responses included formal "exercise therapy or yoga" $(n=21 / 64,32.3 \%)$, and acupuncture $(n=12 / 64,18.5 \%)$. The overall effectiveness of complementary therapies was deemed to be "somewhat effective" in $46 \%$ of participants (Table 4 ).

\section{Cancer treatment modification}

When providers initiated treatment modification, half of respondents indicated they pursued a change in endocrine therapy as their first choice $(33 / 65,50.8 \%)$. Other common responses included dose reduction of endocrine therapy dose $(12 / 65,18.5 \%)$ and treatment breaks $(9 / 65,13.8 \%)$ (Table 3$)$.

\section{Confidence in managing vasomotor symptoms}

When participants were asked to respond to the phrase "I feel confident in managing hot flashes in my patients", 54\% were in agreement with the statement, $20 \%$ disagreed with the statement, and 26\% were neutral (Online Resource 3).

\section{Discussion}

Treatment-related VMS are common in EBC patients, with significant impacts on both quality of life and treatment adherence $[4,5]$. The challenges in addressing VMS in this population include; routinely asking patients about symptoms, health care providers acknowledging the patient's perception of severity, and optimal treatment options. Although most respondents stated that patients were routinely asked about hot flashes, this did include nurses providing their impression of physician practice. Thus, we anticipate that the frequency with which patients are asked about these symptoms is lower, and more in line with the literature, where only $1 / 3^{\text {rd }}$ of patients are routinely asked about these symptoms [3]. Health care providers acknowledged the commonality and severity of VMS and sleep disruptions, while intimacy was felt to be the least bothersome symptom. Providers were more likely to treat VMS in response to direct patient request, concerns of reduced breast cancer treatment adherence, and sleep difficulties.

It was clear that many different interventions were utilised to manage VMS. After lifestyle interventions, the most common pharmacologic strategies utilized were anti-depressants
(73.8\%), in keeping with various trial findings and society guidelines [10-13]. Ninety-one percent of health care providers perceived that anti-depressants were at least "somewhat effective" in managing hot flash symptoms. However, a study of 665 breast cancer patients found that only $45-55 \%$ found these treatments to be effective [3] and adverse events have been reported in up to $80 \%$ of patients on antidepressants [12]. Health care providers were less likely to recommend complementary and over-the-counter interventions. This may be related to lack of familiarity with these treatment modalities; however, this was not assessed in this survey. If VMS remain unmanaged, health care providers reserved modifications to endocrine therapy for later lines of treatment. It is interesting that $54 \%$ of health care providers reported being comfortable managing VMS in their breast cancer patients when overall the treatment appears sub-optimal, with so many potential interventions with limited perceived efficacy..

This survey has several limitations. Firstly, the response rate for our survey was only $17 \%$ compared to past surveys with response rates of $30-50 \%$. This is likely related to the study being conducted during the COVID-19 pandemic, where an increase in email volume and the focus on transitioning to virtual patient care mediums likely impacted response rates. A second limitation of this study includes the surveying of health care providers who may not be directly involved with the prescription of therapies for VMS. While we had intended for two thirds of the study population to be physicians, the balance between nurses and physicians was nearly equivalent in the final dataset. Whether physician or nurse, very few participants responded "I don't know" to questions relating to prescriber practices. Similarly, presenting the data based on provider specialty (i.e. physicians and nurses) resulted in the number of respondents to some questions being so small as to preclude meaningful conclusions to be drawn. As the responses by both physicians and nurses were similar across the survey questions, we therefore felt that combining all health care provider results was more appropriate.

A further limitation was that it was not possible to give response rates for the CANO members as this group includes nurses involved in the care of all tumour types. A survey question that was not included, but may have been helpful, would have been the perceived effectiveness of anti-cancer treatment dose modification strategies, and lifestyle modifications. Moreover, as lifestyle modifications were the most common intervention for health care providers, inquiring about specific recommendations utilized would have been informative. 


\section{Conclusions}

The effective management of VMS is an important component of breast cancer survivorship. However, while multiple possible interventions are available to manage these symptoms, there is limited robust data to guide clinicians in the selection of therapies. Following integration of lifestyle interventions, most clinicians prefer treatment of VMS with anti-depressants, with less uptake of over-the-counter supplement therapy or complementary therapies that may carry fewer side effects. Further randomized clinical trials with patient-focussed outcomes are needed.

Authors' contributions $\mathrm{KC}, \mathrm{SM}, \mathrm{MC}, \mathrm{MA}, \mathrm{LV}, \mathrm{FM}, \mathrm{AP}$, and GL designed the survey and prepared the protocol. LV collected the data and coordinated the study. KC did the statistical analysis and wrote the manuscript. All authors had full access to the data and took responsibility for the integrity of the data and accuracy of the data analysis. All authors were involved in the critical review of the manuscript and approved the final version.

Funding This research did not receive any specific grant from funding agencies in the public, commercial, or not-for-profit sectors. This work was supported by the Rethinking Clinical Trials (REaCT) program platform at the Ottawa Hospital, which is supported by The Ottawa Hospital Foundation and its donors.

Data availability The datasets generated during and/or analysed during the current study are available from the corresponding author on request with approval of the Ontario Cancer Research Ethics Board.

Code availability Not applicable.

\section{Declarations}

Conflict of interest SMG reports receipt of honorarium from Novartis for insights on management of breast cancer patients. BH and MC reports consulting fees from Cornerstone Research, outside the submitted works. All other authors declare no competing interests.

Ethical approval This study was approved by the Ontario Cancer Research Ethics Board (OCREB).

Consent to participate Participation and completion of the survey implied consent to participate.

Consent for publication Participation and completion of the survey implied consent to publication of aggregate survey findings.

\section{References}

1. Howell A, Cuzick J, Baum M, Buzdar A, Dowsett M, Forbes JF, Hoctin-Boes G, Houghton J, Locker GY, Tobias JS, Group AT (2005) Results of the ATAC (Arimidex, Tamoxifen, Alone or in Combination) trial after completion of 5 years' adjuvant treatment for breast cancer. Lancet 365(9453):60-62. https://doi.org/10. 1016/S0140-6736(04)17666-6

2. Pagani O, Regan MM, Walley BA, Fleming GF, Colleoni M, Lang I, Gomez HL, Tondini C, Burstein HJ, Perez EA, Ciruelos E, Stearns V, Bonnefoi HR, Martino S, Geyer CE Jr, Pinotti G, Puglisi F, Crivellari D, Ruhstaller T, Winer EP, Rabaglio-Poretti M, Maibach R, Ruepp B, Giobbie-Hurder A, Price KN, Bernhard J, Luo W, Ribi K, Viale G, Coates AS, Gelber RD, Goldhirsch A, Francis PA, Text, Investigators S, International Breast Cancer Study G (2014) Adjuvant exemestane with ovarian suppression in premenopausal breast cancer. N Engl J Med 371(2):107-118. https://doi.org/10.1056/NEJMoa1404037

3. Fenlon D, Morgan A, Khambaita P, Mistry P, Dunn J, Ah-See ML, Pennery E, Hunter MS, Party NCBCSW (2017) Management of hot flushes in UK breast cancer patients: clinician and patient perspectives. J Psychosom Obstet Gynaecol 38(4):276-283. https:// doi.org/10.1080/0167482X.2017.1350163

4. Stein KD, Jacobsen PB, Hann DM, Greenberg H, Lyman G (2000) Impact of hot flashes on quality of life among postmenopausal women being treated for breast cancer. J Pain Symptom Manage 19(6):436-445. https://doi.org/10.1016/s0885-3924(00)00142-1

5. Chirgwin JH, Giobbie-Hurder A, Coates AS, Price KN, Ejlertsen B, Debled M, Gelber RD, Goldhirsch A, Smith I, Rabaglio M, Forbes JF, Neven P, Láng I, Colleoni M, Thürlimann B (2016) Treatment Adherence and Its Impact on Disease-Free Survival in the Breast International Group 1-98 Trial of Tamoxifen and Letrozole, Alone and in Sequence. J Clin Oncol 34(21):2452-2459. https://doi.org/10.1200/JCO.2015.63.8619

6. ACOG (2014) Practice Bulletin No. 141: management of menopausal symptoms. Obstet Gynecol 123(1):202-216. https://doi. org/10.1097/01.AOG.0000441353.20693.78

7. Clemons M, Goss P (2001) Estrogen and the risk of breast cancer. N Engl J Med 344(4):276-285. https://doi.org/10.1056/NEJM2 00101253440407

8. Hutton B, Hersi M, Cheng W, Pratt M, Barbeau P, Mazzarello S, Ahmadzai N, Skidmore B, Morgan SC, Bordeleau L, Ginex PK, Sadeghirad B, Morgan RL, Cole KM, Clemons M (2020) Comparing Interventions for Management of Hot Flashes in Patients With Breast and Prostate Cancer: A Systematic Review With Meta-Analyses. Oncol Nurs Forum 47(4):E86-E106. https://doi. org/10.1188/20.ONF.E86-E106

9. McGee SF, Vandermeer L, Mazzarello S, Sienkiewicz M, Stober C, Hutton B, Fergusson D, Hilton J, Caudrelier JM, Blanchette P, Clemons M (2019) Physician Survey of Timing of Adjuvant Endocrine Therapy Relative to Radiotherapy in Early Stage Breast Cancer Patients. Clin Breast Cancer 19(1):e40-e47. https://doi. org/10.1016/j.clbc.2018.08.012

10. Boekhout AH, Vincent AD, Dalesio OB, van den Bosch J, Foekema-Töns JH, Adriaansz S, Sprangers S, Nuijen B, Beijnen JH, Schellens JH (2011) Management of hot flashes in patients who have breast cancer with venlafaxine and clonidine: a randomized, double-blind, placebo-controlled trial. J Clin Oncol 29(29):3862-3868. https://doi.org/10.1200/JCO.2010.33.1298

11. Runowicz CD, Leach CR, Henry NL, Henry KS, Mackey HT, Cowens-Alvarado RL, Cannady RS, Pratt-Chapman ML, Edge SB, Jacobs LA, Hurria A, Marks LB, LaMonte SJ, Warner E, Lyman GH, Ganz PA (2016) American Cancer Society/American Society of Clinical Oncology Breast Cancer Survivorship Care Guideline. CA Cancer J Clin 66(1):43-73. https://doi.org/10.3322/ caac.21319

12. Walker EM, Rodriguez AI, Kohn B, Ball RM, Pegg J, Pocock JR, Nunez R, Peterson E, Jakary S, Levine RA (2010) Acupuncture versus venlafaxine for the management of vasomotor symptoms 
in patients with hormone receptor-positive breast cancer: a randomized controlled trial. J Clin Oncol 28(4):634-640. https://doi. org/10.1200/JCO.2009.23.5150

13. Loibl S, Schwedler K, von Minckwitz G, Strohmeier R, Mehta KM, Kaufmann M (2007) Venlafaxine is superior to clonidine as treatment of hot flashes in breast cancer patients-a double-blind, randomized study. Ann Oncol 18(4):689-693. https://doi.org/10. 1093/annonc/mdl478

Publisher's Note Springer Nature remains neutral with regard to jurisdictional claims in published maps and institutional affiliations. 